\title{
Industrial drive based wind simulator for small wind turbine optimisation
}

\author{
S. Paepen MSc*, K. De Koker MSc*, P. Adams MSc* and P. Coussens PhD* \\ * KAHO Sint-Lieven, Department Gent, G. Desmetstraat 1, 9000 Gent, (Belgium)
}

\begin{abstract}
As part of the research effort to optimise a small grid-connected wind turbine, a wind simulator was built. The simulator enables the researcher to simulate any given wind speed as well as a predefined or recorded wind profile. The control variable for the simulation is torque, since the blades in a real situation produce a certain torque on the generator shaft. This generated torque depends on a power curve known as $c_{p}-\lambda$ curve. Apart from the simulated torque an inertia compensation was needed, due to difference in inertia between the real blade situation and the used simulation motor.
\end{abstract}

The simulator was built using nothing but standard industrial components such as an induction motor, industrial drive and NI Labview software.

Index Terms--Computer controlled testbench, Torque control, Wind energy, Wind turbine simulator

\section{NOMENCLATURE}

$\begin{array}{lll}\mathrm{c}_{\mathrm{p}} & - & \text { power coefficient } \\ \mathrm{c}_{\mathrm{T}} & - & \text { torque coefficient } \\ \mathrm{J}_{\mathrm{m}} & - & \text { motor inertia }\left[\mathrm{kg} \cdot \mathrm{m}^{2}\right] \\ \mathrm{J}_{\mathrm{t}} & - & \text { turbine inertia }\left[\mathrm{kg} \cdot \mathrm{m}^{2}\right] \\ \mathrm{P}_{\mathrm{m}} & - & \text { mechanical power }[\mathrm{W}] \\ \mathrm{R} & - & \text { radius of rotor }[\mathrm{m}] \\ \mathrm{T}_{\text {turb }} & - & \text { turbine torque }[\mathrm{N} \cdot \mathrm{m}] \\ \mathrm{T}_{\mathrm{mot}} & - & \text { motor torque }[\mathrm{N} \cdot \mathrm{m}] \\ \mathrm{V}_{\mathrm{w}} & - & \text { wind speed }[\mathrm{m} / \mathrm{s}] \\ \alpha_{\mathrm{m}} & - & \text { angular acceleration }\left[\mathrm{rad} / \mathrm{s}^{2}\right] \\ \beta & - & \text { pitch angle }[\mathrm{rad}] \\ \mathrm{i} & - & \text { gear ratio } \\ \lambda & - & \text { tip speed ratio } \\ \rho & - & \text { air density }\left[\mathrm{kg} / \mathrm{m}^{3}\right] \\ \omega & - & \text { angular speed of turbine }\end{array}$

\section{INTRODUCTION}

When optimising the drive electronics and control strategy for a small wind turbine it is important to be able to test the control strategy in real circumstances. Performing this optimisation in the field was not considered as a feasible method as the real time circumstances are totally unpredictable. Thus, a laboratory set up was needed. With this setup it should be possible to apply the torque generated by wind and blades on the generator shaft without use of the blades.
The construction of this simulator is part of a project to optimise the energy production of small grid-connected wind alternators. This will be done by optimising the topology and control strategy of the energy converters used to make the connection between the alternator and the grid.

The proposed simulator is based on a squirrel cage induction motor and gearbox controlled by an industrial drive. The induction motor is torque controlled, as the torque generated by the turbine depends on its aerodynamic characteristic. This characteristic is defined in the $c_{P}-\lambda$ curve; $c_{P}$ being the power coefficient, and $\lambda$ being the tip speed ratio.

The simulator built in our lab applies a torque profile depending on the set-point "wind speed" and the actual "turbine speed". This profile is equal to the torque profile that would be generated by the turbine blades driven by the wind. The present simulator can generate a mechanical power up to $15 \mathrm{~kW}$, which means that it can test generators up to that power. In practice, generators up to a nominal power of $10 \mathrm{~kW}$ will be tested, giving the opportunity to simulate overload conditions.

\section{AERODYNAMICS: CALCULATION OF THE AVAILABLE MECHANICAL POWER}

The extent to which the available wind energy can be converted into mechanical energy by the blades of a windmill is expressed by the power coefficient $c_{P}$.

The power coefficient $c_{\mathrm{P}}$ can be understood as the efficiency of converting wind power to mechanical power and depends on multiple aerodynamic factors including blade design, the number of blades, airfoil sections, surface finish and angle of attack [1].

Subsequently the available mechanical power can be calculated as:

$$
P_{m}=\frac{1}{2} c_{P} \rho \pi R^{2} v_{w}^{3}
$$

where $c_{P}$ is the power coefficient, $\rho$ the air mass density, $R$ the radius of the blades and $v_{w}$ the wind speed.

The power coefficient is a function of the tip-speed ratio $\lambda$ :

$$
\lambda=\frac{\text { tip speed }}{\text { wind speed }}=\frac{\omega R}{v_{w}}
$$

where $\omega$ is the angular velocity. In Figure 1 can be seen that the rotor will have an optimum speed for every 
wind speed. When the optimum ratio is maintained, a maximum of energy will be extracted from the wind.
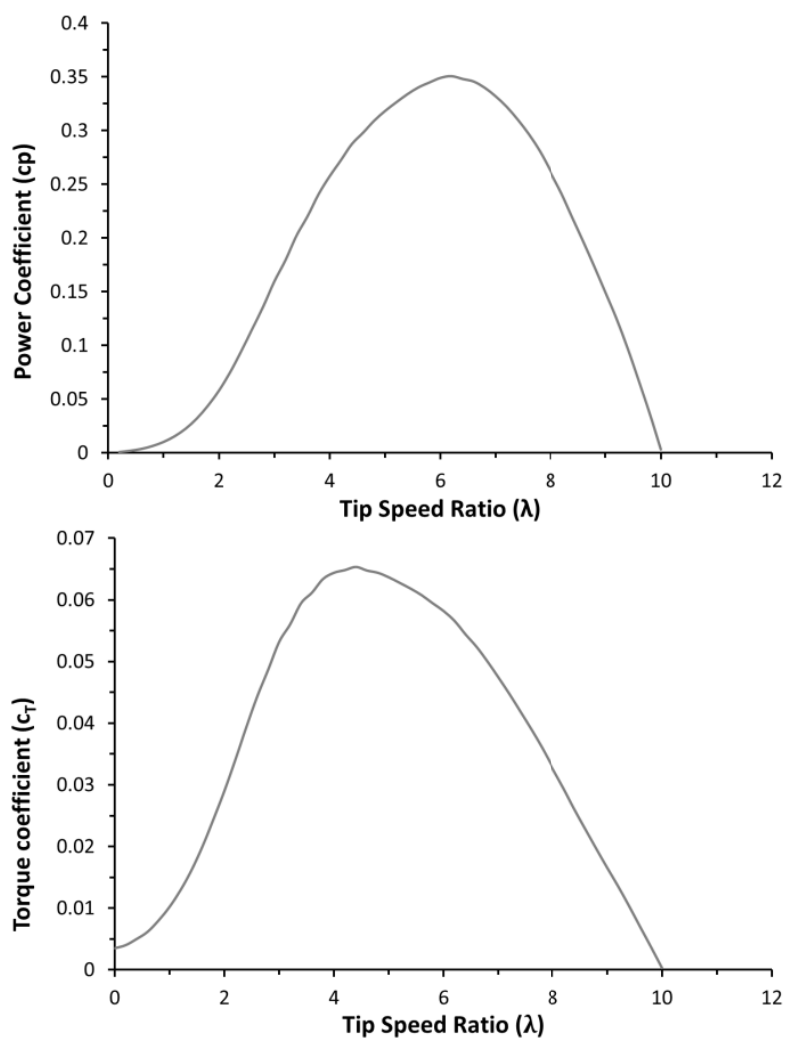

Fig. 1. Curves of $\mathrm{c}_{\mathrm{P}}(\lambda)$ (upper) and $\mathrm{c}_{\mathrm{T}}(\lambda)$ (lower) of a three bladed rotor

In other words, to simulate the mechanical power, one has to take the $c_{P}$ curve into account to control the power. In practice the power will be controlled by means of a torque control, as:

$$
T_{\text {turb }}=\frac{P_{\text {mech }}}{\omega}
$$

Analogous to the expression of the mechanical power, the torque generated by the turbine can be expressed as:

$$
T_{\text {turb }}=\frac{1}{2} c_{T} \rho \pi R^{3} v_{w}^{2}
$$

where $\mathrm{c}_{\mathrm{T}}$ is the torque coefficient [2]. Between $\mathrm{c}_{\mathrm{P}}$ and $\mathrm{c}_{\mathrm{T}}$ the following expression can be found given the expressions (1), (3) and (4):

$$
c_{P}=\lambda c_{T}
$$

The torque control has the additional advantage that the starting torque at $\lambda=0$ is greater than 0 , which can be seen in the right part of Figure 1.

The power and torque coefficients are found by means of wind tunnel experiments or mathematical models and can be described by means of a mathematical general function. The first method will deliver an accurate $c_{P}-\lambda$ curve and different working circumstances can be simulated. The mathematical models such as Blade
Element Momentum theory (BEM) provide accurate data but are generally quite complicated and require a lot of input data. There is also a mathematical function which defines the power coefficient as function of the tip speed ratio $\lambda$ and the pitch angle $\beta$ [2]:

$$
c_{P}=c_{1}\left(c_{2} \frac{1}{\Lambda}-c_{3} \beta-c_{4} \beta^{x}-c_{5}\right) e^{-c_{6} \frac{1}{\Lambda}}
$$

The coefficients $c_{1}$ to $c_{6}$ depend on the turbine rotor type but are described [3] as being equal to: $c_{1}=0.5$, $\mathrm{c}_{2}=116, \mathrm{c}_{3}=0.4, \mathrm{c}_{4}=0, \mathrm{c}_{5}=5$ and $\mathrm{c}_{6}=21$. The parameter $1 / \Lambda$, according to [3] is defined as follows:

$$
\frac{1}{\Lambda}=\frac{1}{\lambda+0.08}-\frac{0.035}{1+\beta^{3}}
$$

\section{CONSTRUCTION OF THE SIMULATOR}

\section{A. Materials}

As the simulator was not the main topic of the research project, the use and combination of standard available equipment seemed the most feasible way of constructing the simulator. Therefore a geared motor and an industrial motor drive were used. The motor is a $15 \mathrm{~kW} 4$ pole induction motor which results in a nominal rotational speed of $350 \mathrm{rpm}$ on the outgoing shaft of the helical gearbox with a 4.22 reduction.

The drive is a Siemens S120 type PM340 with the CU310 processing unit which is used in torque control mode. This drive family offers the possibility to program additional intelligence in a so called DCC-chart (Drive Control Chart).

The control of the drive is done by a standard Windows based personal computer on which Labview is running. Most of the mathematical processing is done by Labview which calculates the set point of the output torque.

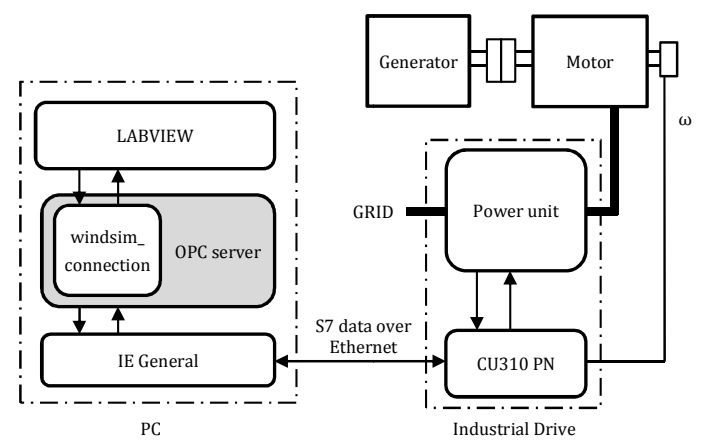

Fig. 2. Schematic of the hardware and data flow

\section{B. Communication}

Ethernet is used as the data carrier for the communication between Labview and the drive. Normally the drive can communicate over Profinet, which supports Isochronous Real Time (IRT) communication with cycles 
times of less than $1 \mathrm{~ms}$ [4]. However no Profinet network card was installed in the computer as the communication speed between PC and drive does not need to be less than $100 \mathrm{~ms}$ as there is no fast loop control needed. The drive communicates with the S7 protocol from Siemens which is also used for PLC communication. Labview does not support this protocol so the use of an OPC (OLE for Process Control) server was necessary.

\section{Torque as a function of the "wind speed" set point and $c_{T}$}

As seen earlier in (6) the $c_{P}-\lambda$ curve can easily be expressed by a mathematical function. Nevertheless if the coefficients $c_{1}$ to $c_{6}$ are not available there is no certainty about the reliability of this curve. There is however an alternate way of acquiring the $c_{P}-\lambda$ curve way which is based on several wind turbine parameters. This method is a mathematical package which is based on the previously mention BEM theory called WT_Perf (Wind Turbine Performance predictor) [5] it is provided by the NWTC (National Wind Technology Center). The method of calculation does not belong to the objective of this paper but uses the lift and drag coefficients of the blade profile. The user inputs other variables such as Reynolds number, calculation method, ....

Based on the provided data by the user, WT_Perf can output the $c_{P}-\lambda$ curve as well as other performance data. The calculated curve can be used to adjust the coefficients of equation (6) to match the wind turbine.

An alternate method to the previous one could be the direct use of the table data provided by WT_Perf in the wind simulator.

The $c_{P}-\lambda$ curve is the key element in the calculation. Once this curve has been defined by means of a table or equation the torque can be easily obtained as a function of the set wind speed, rotor radius and the rotor rotational speed $(1,3)$. All of the above is calculated with Labview with a cycle time of $100 \mathrm{~ms}$.

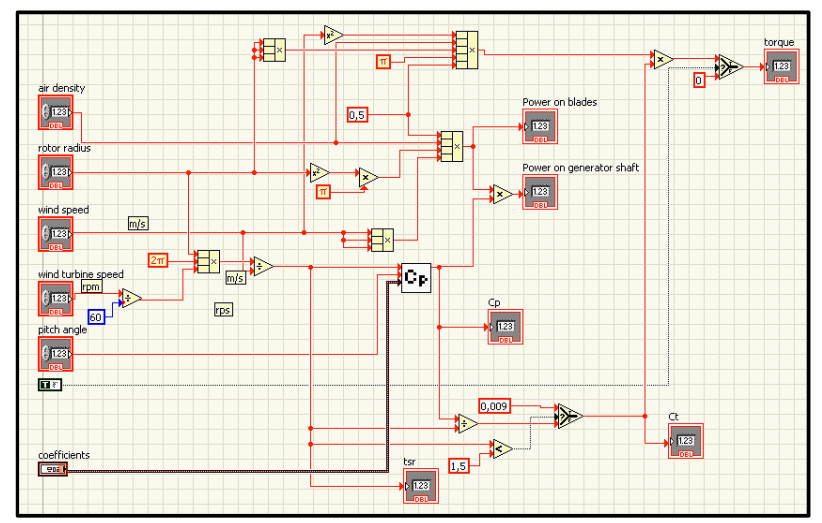

Fig. 3a. Labview turbine model

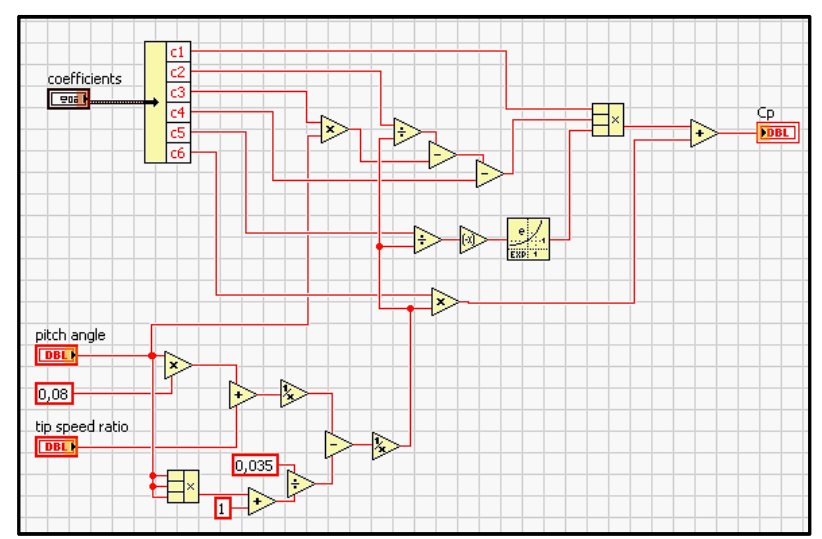

Fig. 4b. Labview cp calculation

\section{Compensation of the inertia}

Next to the simulation of the aerodynamic behavior of the turbine, it is important to take into account the difference in inertia between the rotor blades and the simulator squirrel cage motor and gearbox.
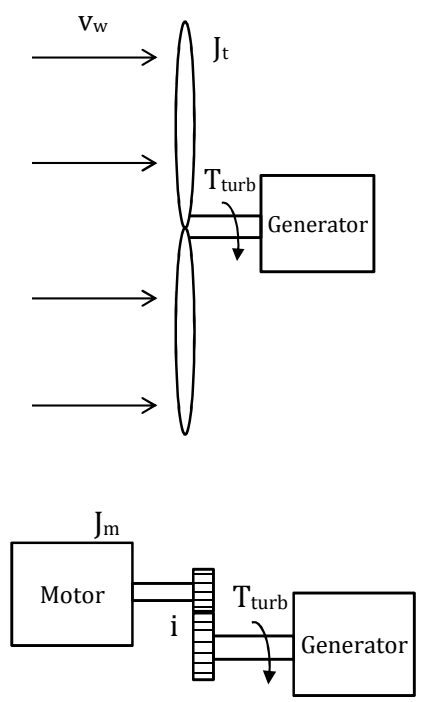

Fig. 5. Inertia relationship between (a) real and (b) simulating situation

This results in the following expression [6]:

$$
T_{m o t}=T_{t u r b}-\left(\frac{J_{t}}{i^{2}}-J_{m}\right) \alpha_{m}
$$

where $T_{\text {mot }}$ is the torque supplied by the motor, $J_{t}$ the moment of inertia of the turbine rotor, $\mathrm{J}_{\mathrm{m}}$ the moment of inertia of the motor, $i$ the gearbox ratio and $\alpha_{m}$ the angular acceleration of the motor. 


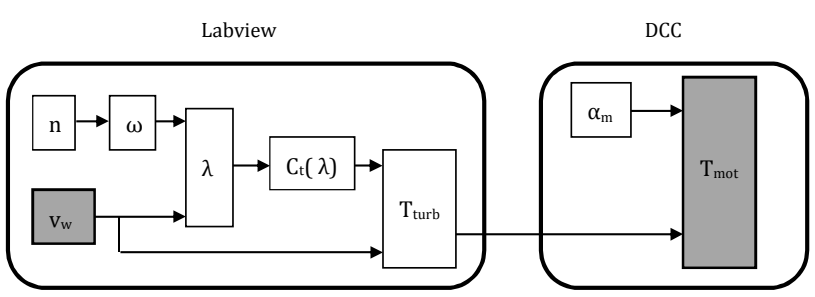

Fig. 6. Flowchart of the torque control using the $\mathrm{c}_{\mathrm{T}}(\lambda)$

Figure 5 shows the simplified Block diagram for the torque calculation. The motor speed $\mathrm{n}$ is measured by an encoder and is converted to angular speed. The wind set point " $\mathrm{V}_{\mathrm{w}}$ " comes from the wind profile to simulate. Both parameters are used to calculate $\lambda$ using Eq. (2). This $\lambda$ is then used in the calculation block to calculate " $\mathrm{c}_{\mathrm{T}}$ ". From this " $\mathrm{T}_{\text {turb" }}$ " is calculated.

However to compensate for the difference in inertia between the simulator and the turbine rotor, this torque should be lowered as per Eq. (8). This is done by differrentiating the measured angular speed to the acceleration $\alpha_{\mathrm{m}}$.

The calculation of the torque compensation is built around the DCC chart of the drive. This is done to obtain a low calculation latency ( $1 \mathrm{~ms}$ cycle time) which is needed for accurate torque compensation.

Now the system will behave as if blades were present on the generator shaft.

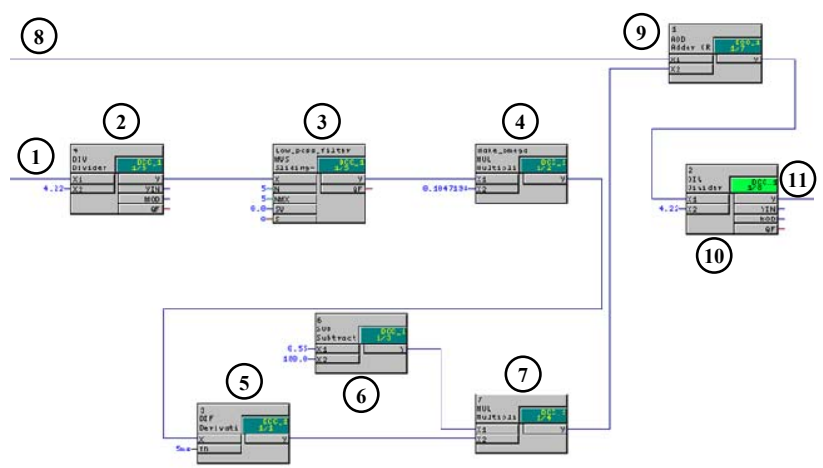

Fig. 7. Torque compensation with a DCC chart

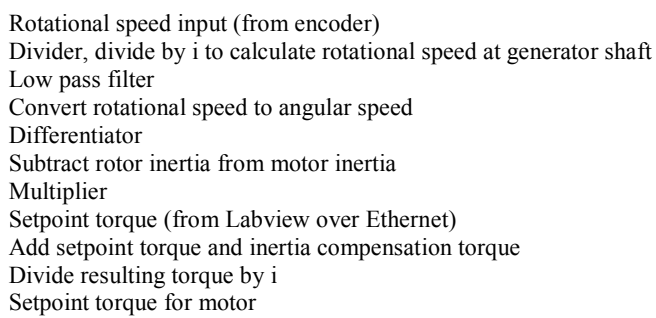

\section{E. The user interface of the simulator}

Besides the mathematical aspects of the simulator, implemented in Labview, the software also offers the possibility to use a graphical user interface. This graphical interface is not only necessary for the control of the windspeed setpoint but should also allow the user to $\log$ all of the necessary data for evaluation afterwards.
Extra features such as monitoring, parameter editing and the use windspeed profiles are also helpful and increase the usability.

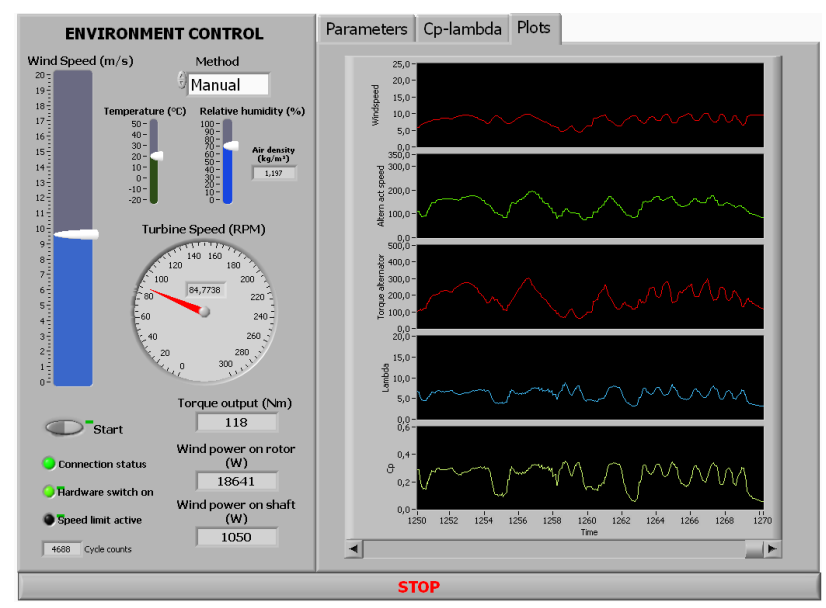

Fig. 7. User interface for the wind simulator with the data logging possibility.

The use of the simulator needed to be as straight forward as possible in order to have a multi-functional system which could be used to test different combinations of turbines and generators.

The next interesting feature is the graphical indication of the actual value of the $c_{P}$. This graph, shown in Figure 8 , gives an immediate idea of the performance of the power tracking strategy of the inverter at the alternator side.

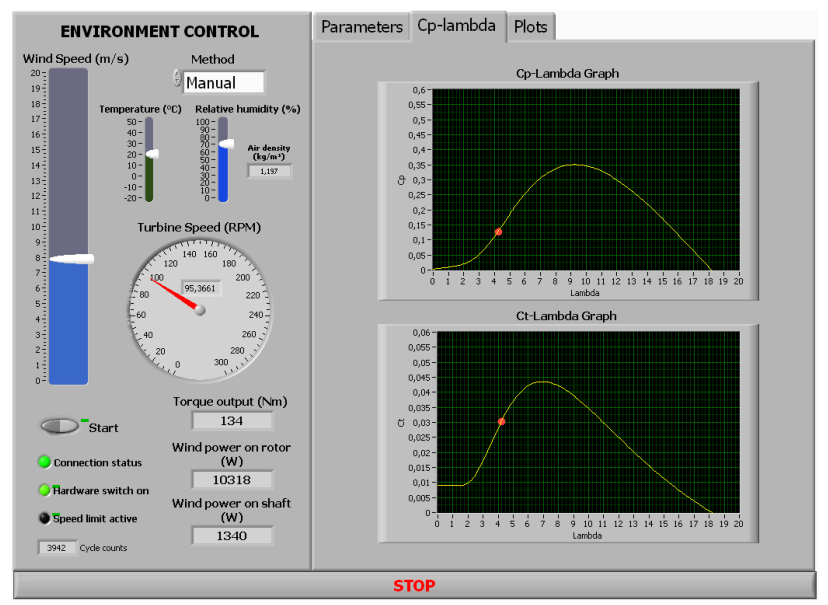

Fig. 8. User interface for the wind simulator with a visualization of the power coefficient

\section{CONCLUSIONS}

The intelligence for the simulation of the aerodynamic characteristics of the rotor blades is realized by Labview while the inertia compensation is calculated by the processor from the industrial drive. Ethernet communication is used to program the drive and to communicate between the user interface and the drive 
during operation.

The key element in the simulator as described is the $c_{p}-\lambda$ curve. This could be obtained through wind tunnel tests but if this is no option mathematical modeling can be an alternative if executed carefully.

The first tests using the simulator as described in the paper generated very promising results. More advanced simulations on turbine generators confirmed and expanded these results. The next step is to install a wind turbine at an experimental site in order to compare the results obtained by the use of the simulator with the onsite results.

In the future the simulator could be expanded with other features such as tower effect, turbulence and surroundings effects, blade roughness and other. The simulator will be used to test several other wind turbines and provide data to manufacturers of small-scale wind turbines.

\section{APPENDIX}

Wind turbine specifications of the experiment setup:

\begin{tabular}{|l|l|}
\hline \multicolumn{2}{|c|}{ Fortis Alizé $-10 \mathrm{~kW}$} \\
\hline rotor diameter & $6,2 \mathrm{~m}$ \\
\hline \# of blades & 3 \\
\hline blade profile & $\mathrm{NLF}-416$ \\
\hline blade pitch & $5^{\circ}$ \\
\hline rotor axis angle & $7^{\circ}$ \\
\hline cut in wind speed & $3 \mathrm{~m} / \mathrm{s}$ \\
\hline rated wind speed & $13 \mathrm{~m} / \mathrm{s}$ \\
\hline
\end{tabular}

\section{ACKNOWLEDGMENT}

This work has been was conducted as a project-based scientific research project by the electrotechnical science group of the Department Gent at the Technical University KAHO Sint-Lieven and was funded by the Flemish government. This research was also partly sponsored by Fortis Wind Energy, Siemens and bvba Vermeulen.

For all questions mail to: PWOwind@kahosl.be

\section{REFERENCES}

[1] De Broe, A.M.; Drouilhet, S.; Gevorgian, V.: A peak power tracker for small wind turbines, IEEE Transactions on Energy Conversion, Vol.14, No.1, 1999

[2] Hau, E.: Wind Turbines: Fundamentals, Technologies, Application, Application, Economics, Springer - Berlin (Germany), 2005.

[3] Lubosny, Z.: Wind Turbine Operation in Electric Power Systems, Springer - Berlin (Germany), 2003.

[4] Pigan, R.; Metter, M.: Automating with PROFINET: Industrial communication based on Industrial Ethernet, Publicis Corporate Publishing - Erlangen (germany), 2006.
[5] Manwell, J.F.; McGowan, J.G.; Rogers, A.L.: Wind Energy Explained, Wiley - England, 2004.

[6] Neammanee, B.; Sirisumrannuku, S.; Chatratana, S.: Development of a Wind Turbine Simulator for Wind Generator Testing, International Energy Journal 8, 2007, pp. 21-28.

[7] De Jong, B.; van der Meulen, R.: De formule Virya, computerberekeningen aan een kleine windmolen, Universiteit Twente, 2007. 